\title{
Determinación de Colinesterasa Eritrocitaria en Trabajadores Agrícolas Expuestos a Plaguicidas Organofosforados y Carbamatos
}

\author{
JENNYFER CUASPUD ${ }^{*}$ *, BEATRIZ VARGAS \\ 'Facultad de Ciencias Químicas, Universidad Central del Ecuador \\ *Correspondencia: jennyferjepano@yahoo.es
}

\section{Resumen}

La enzima colinesterasa constituye el biomarcador de elección para el monitoreo biológico de la población laboral expuesta a plaguicidas organofosforados y carbamatos, los cuales afectan a la salud, por la combinación con la enzima en las terminaciones nerviosas del cerebro y el sistema nervioso impidiendo la transmisión de impulsos nerviosos, provocando una intoxicación. La disminución de los niveles de la enzima colinesterasa en la sangre acarrea varios efectos en el organismo, por esta razón, se ha motivado a realizar esta investigación que permite evaluar el riesgo a la salud por la exposición a plaguicidas inhibidores de colinesterasa. Se realizó la determinación de la actividad de la enzima Colinesterasa Eritrocitaria en 145 muestras biológicas, 95 muestras de agricultores de papa que trabajan con plaguicidas organofosforados y carbamatos inhibidores de la enzima colinesterasa y 50 muestras de un grupo de personas con diversas labores a excepción agrícolas; no expuesto a los plaguicidas para establecer el Valor de Referencia de la zona en la parroquia de Julio Andrade, cantón Tulcán (Carchi-Ecuador). La cuantificación de la actividad de la Colinesterasa Eritrocitaria se realizó empleando el método de Ellman modificado mediante espectrofotometría de luz ultravioleta. El valor promedio de actividad de la Colinesterasa Eritrocitaria en los agricultores que trabajan con plaguicidas organofosforados y carbamatos fue de 3154,99 U/L D.E. +/- 413,23 (valor máximo: $4199 \mathrm{U} / \mathrm{L}$ : valor mínimo: 2320,5 U/L) y en el grupo no expuesto fue de 3625,41 U/L, D.E. +/360,46 3081; valor normal de la Colinesterasa Eritrocitaria de la zona: 3081 - 4745 U/L. El 42 \% de los agricultores que trabajan con los plaguicidas reportaron valores por debajo de los niveles normales de actividad de la Colinesterasa Eritrocitaria. El plaguicida más utilizado por la mayoría de los agricultores de papa (95\%) es Furadán, plaguicida del grupo de los carbamatos.

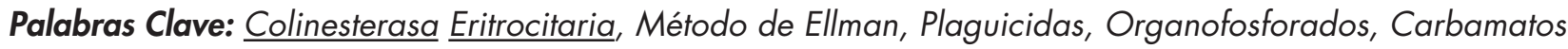

\section{Abstract}

The cholinesterase enzyme, establishes the biomarker of choice for the biological vigilance of the work population exhibit to organophosphates and carbamates pesticides that which affect the human health for the combination with the acetyl cholinesterase enzyme in the nervous ends of brain and Nervous system impeding the transmission of nervous pushes causing a poison, for that reason, to determined of the activity of erythrocyte cholinesterase in 145 biological samples, 95 samples of farmers that work with cholinesterase inhibitor pesticides and, 50 samples of a group non exposed to pesticides for establish the reference value of the zone in people proceeding from Julio Andrade Parish, Tulcan canton (Carchi-Ecuador). Quantification of Erythrocyte Cholinesterase activity was realized applying the Ellman modified method by ultra violet light spectrophotometry. The average value of activity of Erythrocyte Cholinesterase in the farmers that work with 
organophosphates and carbamates pesticides was 3154,99 U/L D.E. +/- 413,26 (maximum value: 4199 $\mathrm{U} / \mathrm{L}$, minimum value:2320,5 U/L) and in the non exposed group was 3625,41 U/L D.E. +/- 360,46; Normal level of activity of erythrocyte cholinesterase in the zone: 3081 - $4745 \mathrm{U} / \mathrm{L}$. The $42 \%$ of the farmers that work with pesticides presented values under normal levels of activity of Erythrocyte Cholinesterase. The pesticide more utilized for the majority of potatoes farmers (95\%) is Furadán (carbamate).

Key Words: Erythrocyte Cholinesterase, Ellman method, Pesticides, Organophosphates, Carbamates

\section{Introducción}

Los agricultores, que trabajan con plaguicidas, están frecuentemente expuestos y pueden sufrir algún tipo de intoxicación por el uso incorrecto de los mismos causando graves secuelas con el pasar del tiempo.

Además, la falta de control de las autoridades sanitarias en el uso de plaguicidas, incluyendo las prácticas inadecuadas de los agricultores como la mezcla de las sustancias con las manos, falta de uso de equipo de protección, equipo de fumigación en mal estado, almacenamiento de plaguicidas dentro de la vivienda, y la eliminación insegura de plaguicidas, contribuyen a una intoxicación por dichas sustancias.

Los plaguicidas son sustancias químicas o mezcla de sustancias destinadas a prevenir, eliminar o controlar cualquier plaga incluyendo los vectores que causan enfermedades humanas o de animales. Se clasifican según el tipo de organismo que controlan, según el grupo químico y según la toxicidad.

Según el grupo químico, consideramos en nuestro estudio a los crganofosforados y carbamatos que afectan al ser humano por la fosforilación de la enzima acetilcolinesterasa en las terminaciones nerviosas del cerebro y el sistema nervioso dando lugar a una disminución de la actividad de la enzima que produce los síntomas de intoxicación.

La acción tóxica de los organofosforados se caracteriza por uniones frecuentemente irreversibles de los radicales fosfatos a los sitios activos de la enzima creando enzimas fosforiladas. El resultado es una pérdida de la acetilcolinesterasa disponible por lo que la terminal sináptica permanece hiperestimulada por un exceso de acetilcolina.

Dependiendo de la actividad de los compuestos organofosforados, a nivel de los receptores muscarínicos o nicotínicos del cuerpo, los signos y síntomas de la intoxicación pueden agruparse, a su vez, en tres síndromes de base colinérgica y son: el Muscarínico, el Nicotínico y el del Sistema Nervioso Central.

Los carbamatos actúan igual que los organofosforados como inhibidores de la colinesterasa, con la única diferencia de que la estabilidad de la unión enzima tóxico es inferior, por lo que se clasifica como inhibidor reversible de la colinesterasa.

Los plaguicidas organofosforados y carbamatos se absorben fácilmente por inhalación, ingestión y a través de la piel.

La Colinesterasa es una enzima del grupo de las esterasas situada en las hendiduras sinápticas, cuya función es hidrolizar a la Acetilcolina un neurotransmisor que mediante la unión a sus receptores, permite que las sinapsis colinérgicas transmitan los impulsos nerviosos. La colinesterasa produce la inactivación de la acetilcolina, con la consiguiente disminución de la transmisión del impulso nervioso. 
Los seres humanos poseen tres tipos de colinesterasa:

- Colinesterasa Eritrocitaria.

- Colinesterasa Cerebral.

- Colinesterasa Plasmática.

La Colinesterasa Eritrocitaria, considerada en nuestro estudio, llamada también Acetilcolinesterasa o Colinesterasa verdadera, es una enzima que hidroliza a su sustrato la Acetilcolina, en forma específica. Se trata de una glicoproteína extracelular con un peso molecular de aproximadamente 80,000 Da que se encuentra presente en los eritrocitos, tejido nervioso, en las sinapsis ganglionares de la estructura neuromuscular, músculo esquelético y placenta.

La presente investigación permitió conocer los niveles de actividad de Colinesterasa Eritrocitaria utilizando un método cinético - espectrofotométrico, modificado de ELLMAN, empleando el ácido 6,6 -ditiodinicotínicoDTNA.

Según el método, la tiocolina producto obtenido de la hidrólisis por acción de la enzima Colinesterasa, reduce al DTNA liberando ácido tionicotínico, permitiendo la determinación de la enzima mediante el monitoreo continuo del aumento de absorbancia a $340 \mathrm{~nm}$ debido a que el pico de absorbancia máxima del ácido tionicotínico se presenta a $340 \mathrm{~nm}$.[1]

El aumento de absorbancia a $340 \mathrm{~nm}$ es directamente proporcional a la actividad de la enzima

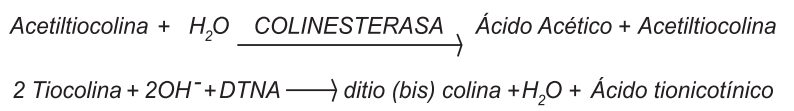

Además, a través de una encuesta ocupacional en la que se consultó variables como sexo, edad, tiempo de exposición, lugar de almacenamiento de plaguicidas, equipo de protección usado, capacitación en el manejo de plaguicidas, tipo de plaguicida utilizado, signos y síntomas clínicos a los agricultores seleccionados, se conoció en cierta forma el grado de relación entre sus niveles de actividad de Colinesterasa Eritrocitaria y algunas de sus características, hábitos y costumbres.

De acuerdo a la técnica de muestreo probabilístico aleatorio simple, el tamaño de la muestra que se investigo se calculó a través del programa EPI INFO donde se obtuvo los siguientes resultados:

\begin{tabular}{cc}
\hline Tamaño Poblacional & 2500 individuos \\
\hline Prevalencia esperada & $50,0 \%$ \\
Peor resultado & $60,0 \%$ \\
Nivel de Confianza & $95,0 \%$ \\
\hline Tamaño de la Muestra & $\mathbf{9 2}$ individuos \\
\hline
\end{tabular}

Fuente: La autora.

Se tomaron al azar 95 muestras de agricultores de papa de ambos sexos para lo cual se tuvo en cuenta los siguientes criterios de elección:

Individuos mayores de 18 años que declararon haber trabajado con plaguicidas en un período no mayor de 20 días antes de la toma de muestra.

No se consideró mujeres embarazadas o en período menstrual, ni individuos que declararon tener una enfermedad controlada o que tomen medicamentos.

Para el grupo control se tomaron 50 muestras de sangre de individuos de ambos sexos mayores de 17 años aparentemente sanos, no expuestos a plaguicidas con labores diferentes a las agrícolas (amas de casa, conductores, obreros, comerciantes, estudiantes, profesionales) de la zona de estudio.

\section{MATERIALES Y MÉTODOS}

\subsection{EQUIPOS:}

- Baño María calibrado a $30^{\circ} \mathrm{C}$

- Espectrofotómetro de luz UV visible.

- Congelador de $-20^{\circ} \mathrm{C}$

\subsection{MUESTRAS:}

Sangre total recogida con heparina o EDTA. La Colinesterasa presente en los eritrocitos es más parecida químicamente a la Colinesterasa presente en el tejido nervioso, de ahí la importancia de su evaluación en sangre total. 


\subsection{REACTIVOS}

Solución amortiguadora de fosfato, 100 mmol/ L, pH 7,6.

- Disolver 2,33 g de $\mathrm{Na}_{2} \mathrm{HPO}_{4}$ y 0,831 $\mathrm{g}$ de $\mathrm{KH}_{2} \mathrm{PO}_{4}$ en $800 \mathrm{ml}$ de agua destilada.

- Ajustar el pH a 7,6 con $\mathrm{NaOH}$ 0,02M y trasvasar a un matraz volumétrico de un litro.

- Aforar hasta la marca con agua destilada y mezclar.

\section{Solución de trabajo.}

- En un matraz volumétrico de un litro colocar: 61,66 $\mathrm{mg}$ de ácido 6,6'ditiodinicotínico (DTNA), 25,8 mg de hidrocloruro de quinidina y $1 \mathrm{ml}$ de Tritón X-100.

- Aforar hasta la marca con solución amortiguadora de fosfatos $(100 \mathrm{mmol} / \mathrm{l}, \mathrm{pH} 7,6)$ y mezclar.

- Almacenar en botella ámbar.

- Esta solución es estable por lo menos durante 6 meses en refrigeración $\left(4 \mathrm{a}-8^{\circ} \mathrm{C}\right)$.

Sustrato, yoduro de acetiltiocolina, $0,5 \mathrm{mmol} / \mathrm{I}$.

Disolver $30,40 \mathrm{mg}$ de yoduro de acetiltiocolina en 10 $\mathrm{ml}$ de agua destilada. Esta solución es estable por lo menos 3 meses en congelación a $-20^{\circ} \mathrm{C}$.

\subsection{MÉTODO}

Antes de realizar el análisis para la determinación de la actividad de la enzima, los reactivos deben encontrarse a temperatura ambiente.

1. Colocar $2,0 \mathrm{ml}$ de la solución de trabajo en un tubo de vidrio.

2. Agregar $5 \mu$ l de muestra (sangre total). Mezclar e incubar a $30^{\circ} \mathrm{C}$ por dos minutos.

3. Agregar $100 \mu \mathrm{l}$ de sustrato. Mezclar, esperar 30 segundos y determinar el cambio de absorbancia por minuto a $340 \mathrm{~nm}$ ajustando previamente el cero de absorbancia con agua destilada.

\subsubsection{CÁlCULOS}

Bajo las condiciones de la prueba, el coeficiente de absortividad milimolar del ácido tionicotínico a $340 \mathrm{nM}$ y $30^{\circ} \mathrm{C}$ es $10,80 \mathrm{~L} \mathrm{mmol}^{-1} \mathrm{~cm}^{-1}$.

\footnotetext{
Acetilcolinesterasa $U / L=\frac{\Delta A / \min \times 2.105 \mathrm{ml}^{-1}}{0,005 \mathrm{ml} \times 10.80 \times 10^{-3} \mathrm{Lmmol}^{-1} \mathrm{~cm}^{-1}}$

\section{RESULTADOS Y DISCUSIONES}

Después del análisis de las 95 muestras tomadas a agricultores de papa que trabajan con plaguicidas inhibidores de la actividad de la enzima Colinesterasa Eritrocitaria (Grupo expuesto) y de las 55 muestras tomadas a personas no expuestas a los plaguicidas (Grupo Control) se observó lo siguiente:

\section{Parámetros estadísticos comparativos} de la actividad de la enzima Colinesterasa Eritrocitaria del grupo de personas no expuestas y del grupo de agricultores expuestos a plaguicidas

Zona Analizada: Parroquia Julio Andrade, Cantón Tulcán.

Número de muestras totales: 145

Cuadro № 1

\begin{tabular}{ccc}
\hline Parámetros & \multicolumn{2}{c}{ Grupo } \\
\hline Número de muestras & 95 & 50 \\
Valor promedio (U/L) & 3154,99 & 3625,41 \\
Valor Máximo (U/L) & 4197,0 & 4745 \\
Valor Mínimo (U/L) & 2320,5 & 3081,0 \\
Desviación estándar & 413,23 & 360,46 \\
\hline Coeficiente de variación (\%) & 0,131 & 0,099
\end{tabular}

Fuente: La autora.

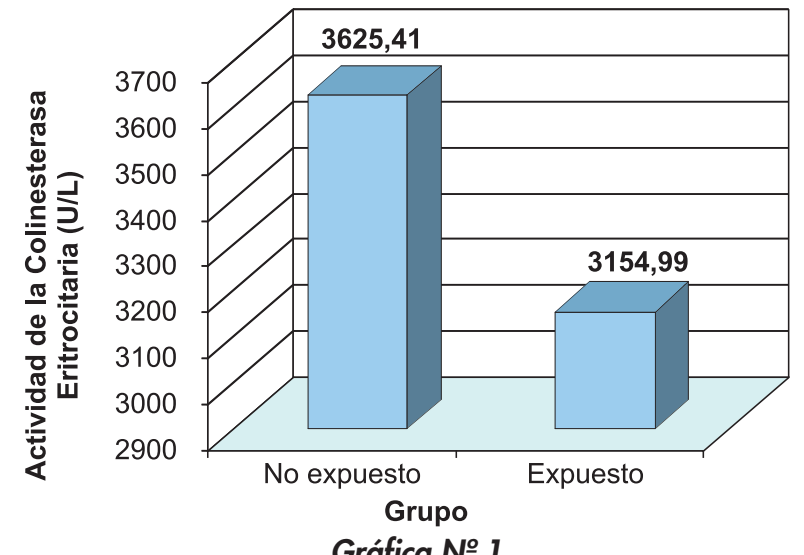

Fuente: La autora.

Debido al amplio rango de valores en los niveles de actividad de la Colinesterasa Eritrocitaria, existe una alta 
variabilidad tanto en el grupo de agricultores expuestos a plaguicidas como en el grupo control; pero, según los coeficientes de variación (C.V.) la variabilidad es mayor en el grupo de los agricultores expuestos (C.V.: 0,131) que en el grupo de control (C.V.: 0,099). (Cuadro № 1) debido a que se tomaron en cuenta, para el grupo expuesto, variables que pueden afectar la medición tales como: edad, tiempo de exposición, signos y síntomas, etc.

Además, para el valor medio de actividad de la Colinesterasa Eritrocitaria de los agricultores expuestos a plaguicidas $(3154,99 \mathrm{U} / \mathrm{L})$ y del grupo de control $(3625,41 \mathrm{U} / \mathrm{L})$ existe una diferencia de medias estadísticamente significativa $(p<0,05)$, valor obtenido a través de la prueba de $t$ de student, indicando que la exposición a plaguicidas Organofosforados y Carbamatos afecta los niveles de actividad de la Colinesterasa Eritrocitaria en los agricultores expuestos.

\section{Distribución de agricultores expuestos a plaguicidas de acuerdo a la normalidad de sus niveles de actividad de la Colinesterasa Eritrocitaria}

\section{Cuadro № 2}

\begin{tabular}{crcc}
\hline $\begin{array}{c}\text { Nivel de actividad de la } \\
\text { Colinesterasa Eritrocitaria (U/L) }\end{array}$ & $\begin{array}{c}\boldsymbol{N}^{\circ} \text { de } \\
\text { personas }\end{array}$ & (\%) \\
\hline \multirow{2}{*}{$\begin{array}{c}\text { DISMINUIDO } \\
\text { NORMAL }\end{array}$} & $(2320,5-3081) \mathrm{U} / \mathrm{L}$ & 42 & 44,21 \\
& $(3081-4745) \mathrm{U} / \mathrm{L}$ & 53 & 55,79 \\
\hline & TOTAL & 95 & 100.0 \\
\hline
\end{tabular}

Fuente: La autora.

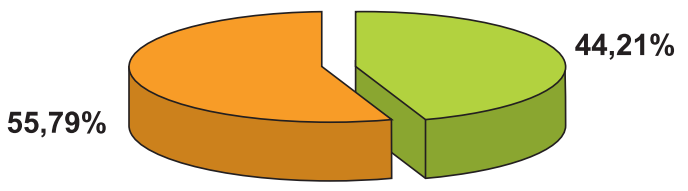

DISMINUIDO

$\square$ NORMAL
El $44,21 \%$ del total de agricultores expuestos, 142 casos) tienen sus niveles de actividad de Colinesterasa Eritrocitaria por debajo de los valores de referencia de la zona (3081 - 4745) U/L mientras que el 55,79\% (53 casos) de agricultores tienen sus niveles dentro del valor de referencia. (Cuadro № 2).

Niveles promedio de la actividad de la Colinesterasa Eritrocitaria y distribución de agricultores expuestos a plaguicidas según el sexo

Valores Normales: $3081-4745 \mathrm{U} / \mathrm{L}$

Cuadro № 3

\begin{tabular}{cccc}
\hline SEXO & $\begin{array}{c}\mathbf{N}^{\circ} \text { de } \\
\text { personas }\end{array}$ & (\%) & $\begin{array}{c}\text { Nivel promedio de } \\
\text { actividad de la } \\
\text { Colinesterasa } \\
\text { Eritrocitaria (U/L) }\end{array}$ \\
\hline Hombres & 87 & 90,52 & 3182,80 \\
Mujeres & 8 & 9,48 & 3270,15 \\
Total & 95 & 100,0 & \\
\hline
\end{tabular}

Fuente: La autora.

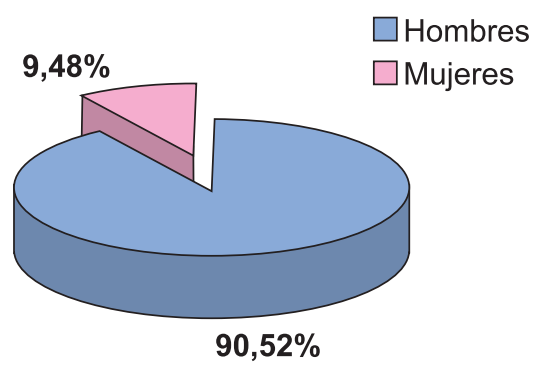

Gráfica № 3

Aunque el valor medio de actividad de la Colinesterasa Eritrocitaria en mujeres $(3270,15 \mathrm{U} / \mathrm{L})$ es mayor que el valor medio de los hombres (3182,80 U/L). (Cuadro № 3), no existe una diferencia de medias estadísticamente significativa de acuerdo al nivel de significancia $(p<0,05)$, por lo que no puede afirmarse que las mujeres tienen niveles de actividad superiores al de los hombres. 
Niveles promedio de la actividad de la Colinesterasa Eritrocitaria y distribución de agricultores expuestos a plaguicidas según la edad

Cuadro № 4

\begin{tabular}{cccc}
\hline $\begin{array}{c}\text { Edad } \\
\text { (años) }\end{array}$ & $\begin{array}{c}\mathbf{N}^{\mathbf{2}} \\
\text { personas }\end{array}$ & $\mathbf{( \% )}$ & $\begin{array}{c}\text { Nivel promedio de } \\
\text { actividad de la } \\
\text { Colinesterasa } \\
\text { Eritrocitaria (U/L) }\end{array}$ \\
\hline $18-24$ & 13 & 13,68 & $3207,89 \pm 462,43$ \\
$25-31$ & 15 & 15,79 & $2993,62 \pm 424,81$ \\
$32-38$ & 18 & 18,95 & $3199,24 \pm 264,35$ \\
$39-45$ & 18 & 18,95 & $3281,47 \pm 492,38$ \\
$46-52$ & 19 & 20,00 & $3147,82 \pm 439,75$ \\
$53-59$ & 8 & 8,42 & $3099,76 \pm 432,38$ \\
$60-66$ & 4 & 4,21 & $3045,93 \pm 147,58$ \\
\hline Total & 95 & 99,99 & \\
\hline
\end{tabular}

Fuente: La autora.

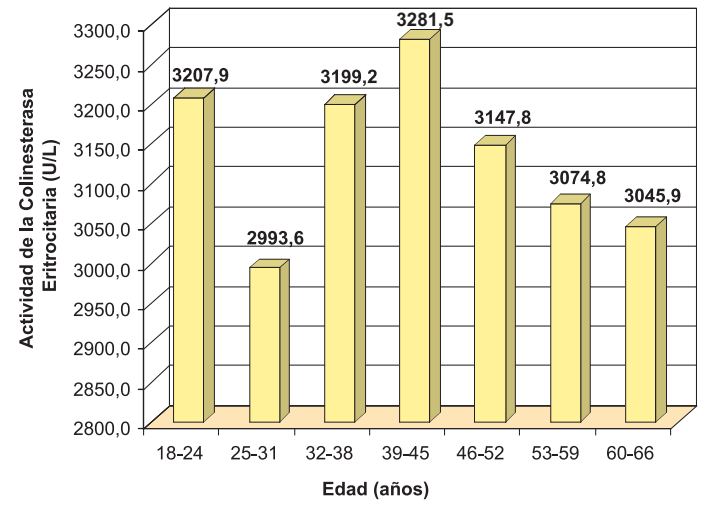

Gráfica № 4

En cuanto al nivel promedio de actividad de Colinesterasa Eritrocitaria según la edad de los agricultores se observó un cierto grado de correlación, es decir, a mayor edad, menor nivel promedio a excepción del intervalo de edad de 25 a 31 años en que se evidenció una mayor disminución del nivel de actividad de la enzima 2993,6 U/L con respecto al intervalo de 60 a 66 años $3045,9 \mathrm{U} / \mathrm{L}$ y de igual forma, se observó con el intervalo de edad de 32 a 38 años en el que se evidenció una ligera disminución del nivel de actividad de la enzima con respecto al intervalo de 39 a 45 años. La explicación de este hecho se debe a que los agricultores de papa, cuyas edades comprenden entre dichos intervalos, son la mayor fuerza laboral por lo que tienen una mayor exposición a los plaguicidas organofosforados y carbamatos.

En agricultores con edades de 18 a 24 años ( 13 casos) el nivel promedio fue de $3207,89 \mathrm{U} / \mathrm{L}$, mientras aquellos con edades mayores a los 60 años ( 4 casos) el nivel promedio fue de 3045,93 U/L (Cuadro № 4). Esto se debe que a mayor edad, mayor es el tiempo de exposición y en consecuencia menor es el nivel de actividad de Colinesterasa Eritrocitaria.

Se apreció que el intervalo de edad de mayor incidencia fue entre 25 a 31 años, se encontraron 15 casos (Cuadro № 4).

\section{Niveles promedio de actividad de la Colinesterasa Eritrocitaria y distribución de agricultores expuestos a plaguicidas según tiempo de ocupación}

Cuadro № 5

\begin{tabular}{cccc}
\hline $\begin{array}{c}\text { Tiempo de } \\
\text { ocupación } \\
\text { (años) }\end{array}$ & $\begin{array}{c}\mathbf{N}^{\circ} \\
\text { personas }\end{array}$ & (\%) & $\begin{array}{c}\text { Nivel promedio de } \\
\text { actividad de la } \\
\text { Colinesterasa } \\
\text { Eritrocitaria (U/L) }\end{array}$ \\
\hline 1 a 8 & 15 & 17,85 & $3257,2 \pm 388,15$ \\
9 a 16 & 29 & 34,52 & $2977,3 \pm 237,71$ \\
17 a 24 & 16 & 19,05 & $3062,2 \pm 51,73$ \\
25 a 32 & 16 & 19,05 & $2981,3 \pm 534,95$ \\
33 a 40 & 7 & 8,33 & $3055,1 \pm 410,02$ \\
41 o más & 1 & 1,19 & 2895,8 \\
\hline Total & 84 & 99,99 & \\
\hline
\end{tabular}

Fuente: La autora.

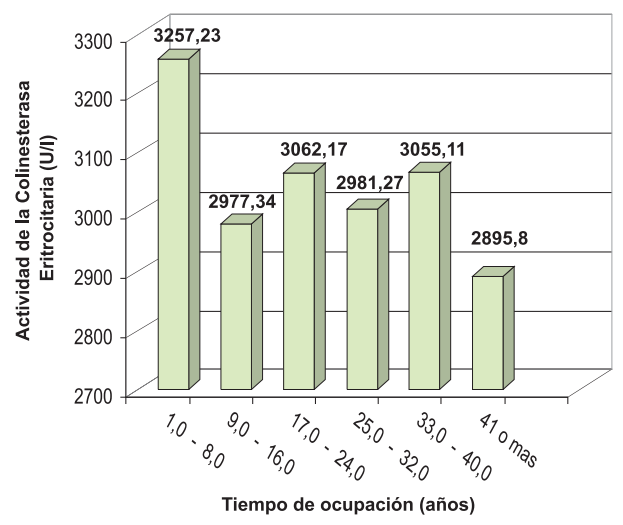

Gráfica № 5 
En cuanto al nivel promedio de actividad de la Colinesterasa Eritrocitaria según el tiempo de ocupación de los agricultores se presentó que a partir de los 9 años de tiempo de ocupación, los niveles promedio de actividad de la Colinesterasa Eritrocitaria disminuyen por debajo de los niveles normales de la actividad de la enzima, es decir, que a mayor tiempo de ocupación menor es el nivel promedio.

En agricultores con tiempo de ocupación menor de 9 años ( 15 casos) el nivel promedio fue de $3257,2 \mathrm{U} / \mathrm{L}$, mientras que en aquellos con tiempo de ocupación mayor de 9 años (80 casos) el nivel promedio fue de 2994,3 U/L. (Cuadro № 5).

\section{Niveles promedio de actividad de la Colines- terasa Eritrocitaria y distribución de agricul- tores expuestos a plaguicidas según el lugar de almacenamiento de los plaguicidas}

Cuadro № 6

\begin{tabular}{ccccc}
\hline $\begin{array}{c}\text { Lugar de } \\
\text { almacena- } \\
\text { miento }\end{array}$ & $\begin{array}{c}\boldsymbol{N}^{\circ} \text { de } \\
\text { personas }\end{array}$ & (\%) & $\begin{array}{c}\text { Nivel promedio de } \\
\text { actividad de la } \\
\text { Colinesterasa } \\
\text { Eritrocitaria (U/L) }\end{array}$ \\
\hline $\begin{array}{c}\text { Área } \\
\text { Exclusiva }\end{array}$ & 52 & 54,74 & $3212,75 \pm 471,32$ \\
$\begin{array}{c}\text { Dentro de } \\
\text { la casa }\end{array}$ & 5 & 5,26 & 3066,9 & $\pm 313,94$ \\
$\begin{array}{c}\text { Fuera de } \\
\text { la casa }\end{array}$ & 38 & 40,00 & $3180,4 \quad \pm 415,88$ \\
\hline Total & 95 & 100 & & \\
\hline
\end{tabular}

Fuente: La autora.

$39,18 \%$

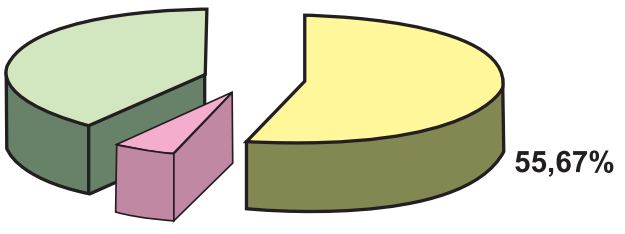

$5,15 \%$
El valor promedio de actividad de la Colinesterasa Eritrocitaria de agricultores que almacenan los plaguicidas dentro de la casa 3066,9 U/L es menor que el de los agricultores que almacenan fuera de la casa $3180,4 \mathrm{U} / \mathrm{L}$, mientras que los agricultores que almacenan los plaguicidas en un área exclusiva 152 casos) el valor promedio de la actividad de la enzima $3212,75 \mathrm{U} / \mathrm{L}$ es mayor que de aquellos que almacenan fuera de la casa. (Cuadro № 6).

Es posible que estos datos se deban a que los agricultores que almacenan los plaguicidas lejos del lugar donde habitan están menos expuestos a estos, cuando no están aplicando los plaguicidas.

\section{Distribución de los agricultores expuestos a plaguicidas según los signos y síntomas presentados}

En cuanto a los signos y síntomas más resaltantes mencionados por los agricultores tenemos:

Síntomas Muscarínicos: El 69,39\% (68 casos) de ellos presentaba ardor ocular siendo este el grupo de mayor incidencia; $37,76 \%$ (37 casos) de ellos presentaba visión borrosa; $31,63 \%$ (31 casos) de ellos presentaba sudoración; 31,63 \% (33 casos) de ellos presentaba dolor abdominal; $18,37 \%$ (18 casos) de ellos presentaba dificultad respiratoria, siendo este el grupo de menor incidencia. (Gráfica № 7).

Síntomas Nicotínicos: El 57,14 \% (56 casos) de ellos presentaba mareos, siendo este el grupo de mayor incidencia; 54,08 \% (53 casos) de ellos presentaba cefaleas; $50 \%$ (49 casos) de ellos presentaban debilidad, cansancio o fatiga; $15,31 \%$ (15 casos) de ellos presentaban calambres, siendo este el grupo de menor incidencia. (Gráfica № 8).

Síntomas Neurológicos: El 40,82 \% (40 casos) de ellos presentaba somnolencia, siendo este el grupo de mayor incidencia; $20,41 \%$ (20 casos) de ellos presentaba irritabilidad; $15,31 \%$ (15 casos) de ellos presentaban calambres; $10,2 \%$ (10 casos) de ellos presentaba confusión; 7,1 \% depresión, ansiedad, siendo este el grupo de menor incidencia. (Gráfica № 9).

Debe mencionarse aquí que si bien estas variables juegan un papel primordial en el diagnóstico de una 
intoxicación aguda y crónica, no sucede lo mismo en una intoxicación sub - clínica o sub - aguda como la llaman algunos autores, los síntomas son muy relativos y pueden ser enmascarados por cuadros tan comunes como lumbalgias por el esfuerzo, estado gripal, edad, estrés, etc.

Distribución de los agricultores expuestos a plaguicidas según los signos y síntomas Muscarínicos presentados

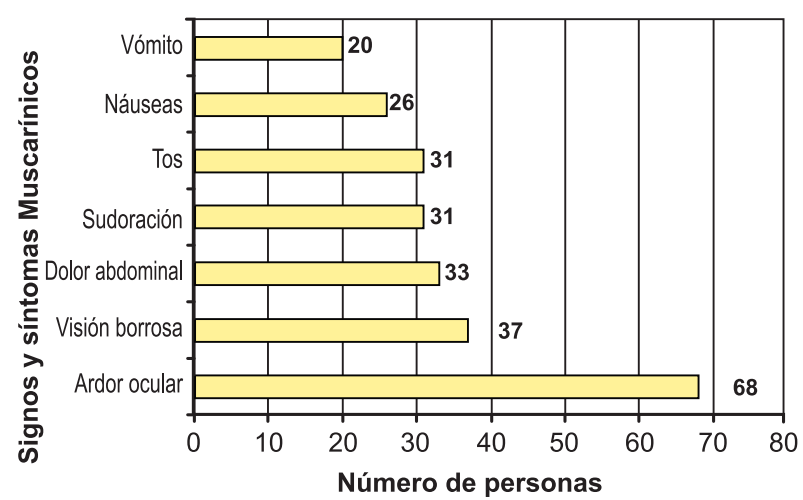

Gráfica № 7

Distribución de los agricultores expuestos a plaguicidas según los signos y síntomas Nicotínicos presentados

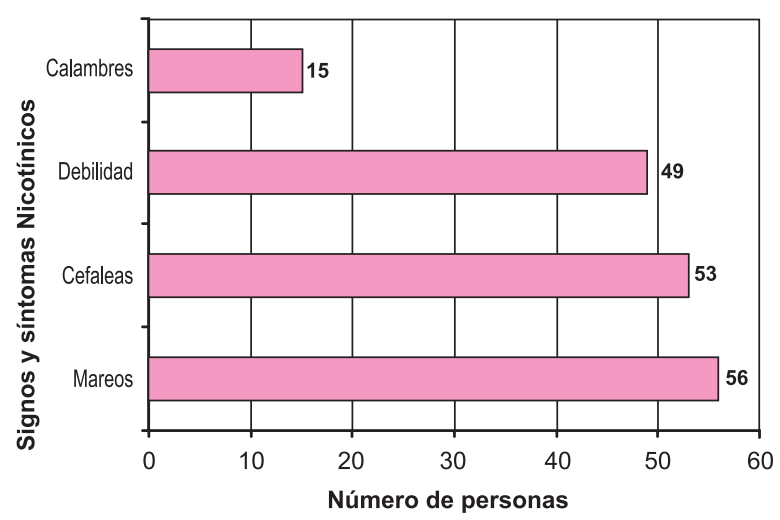

Gráfica № 8
Distribución de los agricultores expuestos a plaguicidas según los signos y síntomas Neurálgicos presentados

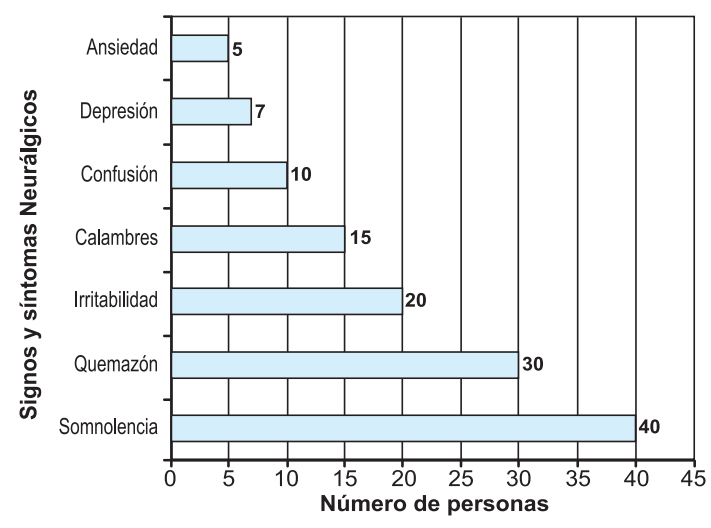

Gráfica № 9

Distribución de agricultores expuestos a plaguicidas según el elemento de protección que utiliza al fumigar

Cuadro № 7

\begin{tabular}{ccc}
\hline $\begin{array}{c}\text { Equipo de } \\
\text { protección }\end{array}$ & $\begin{array}{c}\text { Número de } \\
\text { personas }\end{array}$ & $\begin{array}{c}\text { Porcentaje } \\
\mathbf{( \% )}\end{array}$ \\
\hline Adecuado & 8 & 8,42 \\
Inadecuado & 67 & 70,53 \\
Ninguno & 20 & 21,05 \\
\hline TOTAL & 95 & 100 \\
\hline
\end{tabular}

Fuente: La autora.

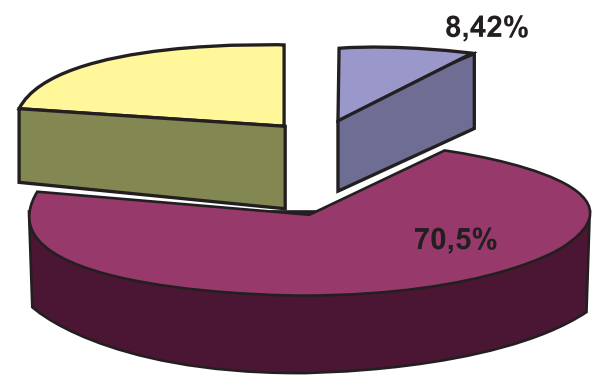

$\square$ ADECUADO $\square$ INADECUADO $\square$ NINGUNO

Gráfica № 10 


\section{Cuadro № 8}

\begin{tabular}{lcc}
\hline \multicolumn{1}{c}{$\begin{array}{c}\text { Elementos de protección } \\
\text { personal }\end{array}$} & $\begin{array}{c}\boldsymbol{N}^{\circ} \text { de } \\
\text { personas }\end{array}$ & (\%) \\
\hline Botas & 4 & 4,1 \\
Botas, gafas y pañuelo & 2 & 2,0 \\
Botas y gorra & 31 & 31,6 \\
Botas y guantes & 9 & 9,2 \\
Botas, guantes y pañuelo & 4 & 4,1 \\
Botas, guantes y gorra & 5 & 5,1 \\
Botas, guantes, gorra y gafas & 2 & 2,0 \\
Botas, guantes, gorra, mascarilla y overol & 5 & 5,1 \\
Botas, guantes, gorra, mascarilla y delantal & 3 & 3,1 \\
Botas, guantes, gorra y amarro & 2 & 2,0 \\
Botas, gorra y mascarilla & 5 & 5,1 \\
Botas, gorra y amarro & 2 & 2,0 \\
Botas, gorra, amarro y delantal plástico & 2 & 2,0 \\
Amarros & 2 & 2,0 \\
Ninguno & 20 & 20,4 \\
\hline TOTAL & 98 & 100,0 \\
\hline
\end{tabular}

Fuente: La autora.

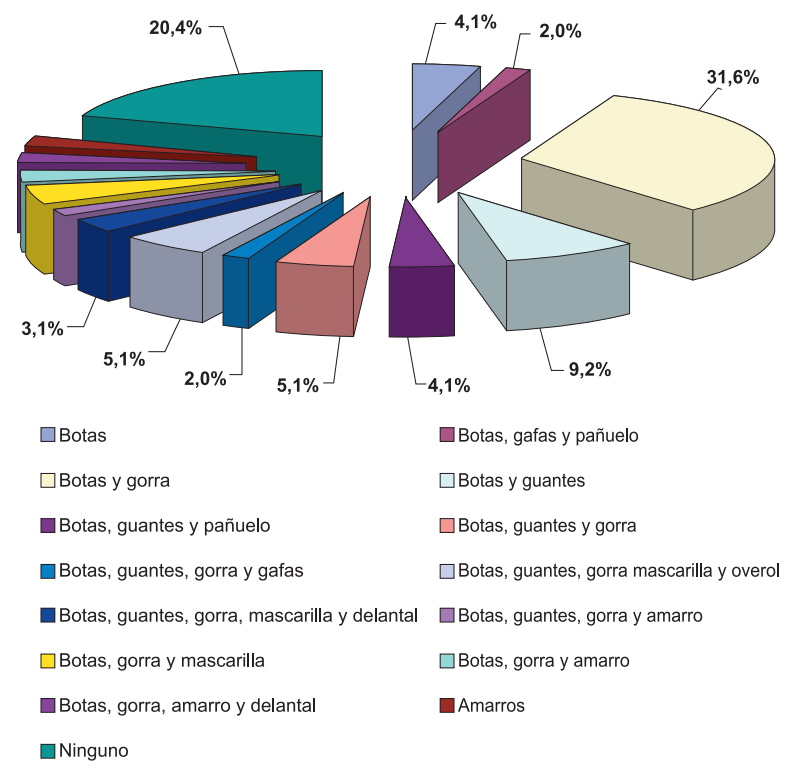

Gráfica № 11
Se observó también en cuanto al uso de equipo de protección personal al momento de fumigar que tan sólo el 8,42 \% (8 casos) usaban el equipo adecuado; el $70,53 \%$ (67 casos) usaban el equipo inadecuado y el 21,05 (20 casos) no utilizaban ningún equipo. (Cuadro № 7$)$.

Se evidenció que $8,2 \%$ (8 casos) de ellos trabajaban con el equipo de protección personal completo (botas, guantes, gorra, mascarilla, delantal u overol) siendo el grupo de menor incidencia; el 3,6 \% (31 casos) de ellos trabajan con el equipo de protección incompleto (botas y gorra) siendo este el grupo de mayor incidencia; el $9,2 \%$ (9 casos) utilizaba botas y guantes; el 5,1 \% (5 casos) utilizaba bota,s guantes y gorra y el 20,4 \% (20 casos) no utilizaba ningún equipo. (Cuadro № 8).

El poco cuidado que tenían los agricultores al momento de realizar la aplicación, explicaría el bajo nivel de actividad de Colinesterasa Eritrocitaria en sus muestras de sangre.

\section{Distribución de los agricultores expuestos a plaguicidas según el tipo de plaguicida utilizado}

Cuadro № 9

\begin{tabular}{cccc}
\hline $\begin{array}{c}\text { Plaguicida } \\
\text { utilizado }\end{array}$ & $\begin{array}{c}\text { Número de } \\
\text { personas }\end{array}$ & $\%$ & $\begin{array}{c}\text { Tipo de } \\
\text { plaguicida }\end{array}$ \\
\hline Furadán & 95 & 96,9 & CB \\
Eltra & 69 & 70,4 & CB \\
Monitor & 45 & 45,9 & OP \\
Curacrón & 42 & 42,9 & OP \\
Curzate & 14 & 14,3 & CB \\
Dithane & 11 & 11,2 & CB \\
Lorsvan & 9 & 9,2 & OP \\
Curater & 7 & 7,1 & CB \\
Manzate & 7 & 7,1 & CB \\
Orthene & 5 & 5,1 & OP \\
\hline Vidate & 3 & 3,1 & OP \\
\hline
\end{tabular}

Fuente: La autora. 


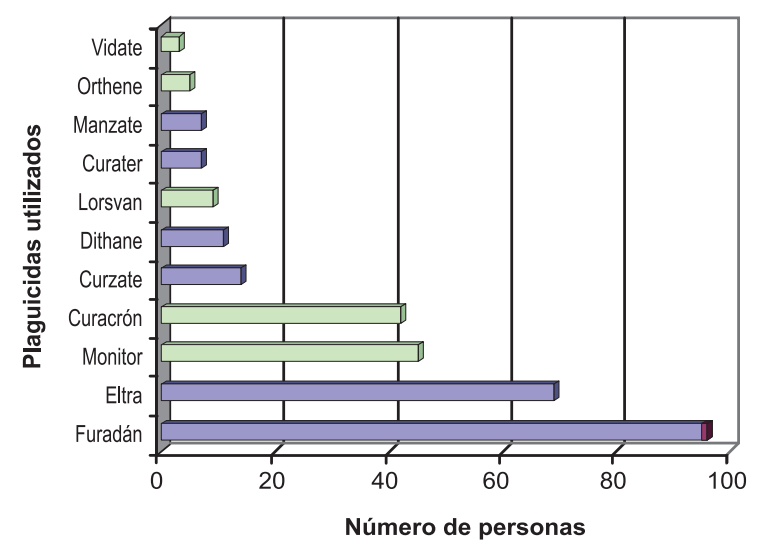

Gráfica № 12

En cuanto al tipo de plaguicida utilizado: el 96,9 \% (95 casos) de ellos utilizan Furadán que es un plaguicida del grupo de los Carbamatos siendo este el de mayor incidencia; 70,4 \% (69 casos) de ellos utilizan Eltra que es un plaguicida del grupo de los Organofosforados; 45,9\% (45 casos) de ellos utilizan Monitor que es un compuesto Organofosforado y en menor proporción utilizan Carbamatos como Curzate (14,3\%), Dithane $(11,2 \%)$, etc. Debe señalarse que la mayoría de los agricultores mezclan los plaguicidas formando cócteles sin conocer su principio activo. (Cuadro № 9).

\section{Distribución de los agricultores expuestos a plaguicidas según su capacitación sobre manejo seguro de plaguicidas}

Cuadro № 10

\begin{tabular}{ccc}
\hline Manejo seguro & $\mathbf{N}^{\circ}$ de personas & (\%) \\
\hline SI & 22 & $23,15 \%$ \\
NO & 73 & $76,85 \%$ \\
\hline TOTAL & 95 & 100 \\
\hline
\end{tabular}

Fuente: La autora.

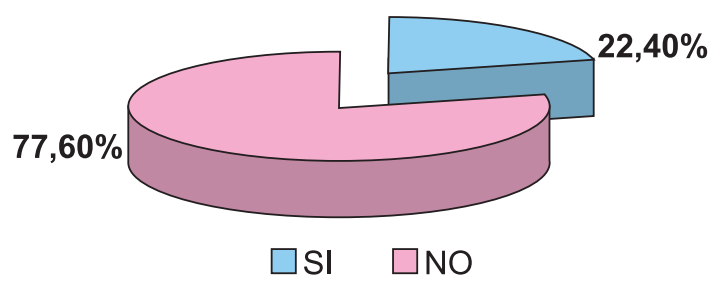

Gráfica № 13
Finalmente se evidenció que el 23,15\% (22 casos) de los agricultores han recibido capacitación sobre el manejo seguro de plaguicidas, mientras que el $76,85 \%$ (73 casos) no han recibido debido a la falta de interés por parte de las autoridades y de los mismos agricultores que al no estar motivados no son partícipes del cuidado de su salud y mejoramiento de la producción de papa con el cuidado al medio ambiente. (Cuadro № $10)$.

\section{CONCLUSIONES}

4.1 De las 95 muestras tomadas a trabajadores agrícolas expuestos a plaguicidas Carbamatos y Organofosforados de la parroquia Julio Andrade del cantón Tulcán se encontró que un $42 \%$ de ellos presentaron niveles de actividad de la Colinesterasa Eritrocitaria por debajo de los valores normales.

4.2 Aunque no se evidenció una marcada disminución de los valores de la actividad de la Colinesterasa Eritrocitaria en el grupo expuesto, la mayoría de agricultores de papa presentaron los signos y síntomas que caracteriza una sobreexposición a plaguicidas y probable intoxicación crónica.

4.3 El nivel promedio de actividad de la Colinesterasa Eritrocitaria de los agricultores de papa expuestos a plaguicidas Organofosforados y Carbamatos fue de 3154,99 U/L por debajo del nivel promedio del grupo no expuesto que fue de $3625,41 \mathrm{U} / \mathrm{L}$, por lo que los compuestos inhibidores de la enzima (Organofosforados y Carbamatos) presentes en los productos que ellos utilizaban, afectó el nivel de actividad de dicha enzima, confirmándose la hipótesis propuesta.

4.4 El nivel promedio de actividad de la enzima Colinesterasa Eritrocitaria del grupo de personas no expuesto a plaguicidas fue de $3625,41 \mathrm{U} / \mathrm{L}$ (valor máximo: $4745 \mathrm{U} / \mathrm{L}$; valor mínimo: 3081 $\mathrm{U} / \mathrm{L}$ ) por lo que, el valor de referencia de la zona de estudio fue de 3081 a $4745 \mathrm{U} / \mathrm{L}$.

4.5 Según la encuesta ocupacional realizada a cada trabajador, el plaguicida más utilizado por los trabajadores agrícolas en el cultivo de papa fue el Furadán (del grupo de los Carbamatos) en un $95 \%$ de los casos; sin embargo, no se evidenció el efecto que produce sobre la actividad de la enzima debido a que cada muestra fue procesada 
a las 15 horas siguientes concluyendo que la medición de la Colinesterasa Eritrocitaria no es un marcador adecuado para la intoxicación por plaguicidas Carbamatos.

4.6 A través de una charla de información sobre el manejo seguro de plaguicidas se incentivó a los agricultores de papa a utilizar elementos de protección personal alternativos al momento de fumigar, y se logró comprometer a las autoridades sanitarias de la zona a realizar capacitaciones continuas a los agricultores, regular los productos de venta en almacenes agrícolas y sobre todo proveer a estos almacenes de equipos de protección personal adecuados y de bajo costo para los trabajadores agrícolas.

\section{Referencias}

1. Barrera, V.; Escudero, L.; Norton, G. y Alwang, J. 2004. Encontrando salidas para reducir los costos y la exposición a plaguicidas en los productores de papa: Experiencia de la intervención en la provincia del Carchi, Ecuador. INIAP, IPM- CRSP, CROPLIFE, FAO. Quito, Ecuador. Pág. 10 a 18.

2. Comba, Pietro, Harari, Raul, El ambiente y la salud, Epidemiología ambiental, 1ra edición, ediciones ABYA - YALA, Quito Ecuador, 2004, Pág. 63 a 77.

3. García Fernández, Juan Carlos.- "Plaguicidas" ("El laboratorio en la Clínica") - Editorial Médica Panamericana, Buenos Aires-1.985Pág.1.323 a 1.341.

4. Henry, Richard, Química Clínica, bases y principios, Edit jims, Barcelona, España, 1969, Pág. 901 - 902,600 - 608.

5. INEN, Asociación de la Industria de Protección de Cultivos y Salud Animal, Normas INEN referentes a plaguicidas, primera edición, Quito - Ecuador, 1992, Pág. 85-86

6. INEN, Norma Ecuatoriana obligatoria en Plaguicidas, Clasificación, Nombres comunes, comerciales y químicos, Quito - Ecuador, 1990, Pág. 1 a 60.

7. Vademecum Agrícola Edifarm, Tercera edición, Editorial EDITHAR, Quito - Ecuador, 1994, Pág. 101 a 392

8. Varios Autores, Manual De Técnicas Analíticas En El Laboratorio de Toxicología, Editorial LEDA, Buenos Aires, Argentina, capítulo 7, PESTICIDAS.

9. Pineda, B, Alvarado, E, Canales, F. Metodología de la Investigación, Manual para el desarrollo de personal de salud, 2da edición, Serie PALTEX, OPS, 1994, Pág. 80-87

10. Vargas Beatriz, Ensayos de Toxicología General, Clínica, Farmacéutica y forense de la Facultad de Ciencias Químicas de la Universidad Central del Ecuador. Quito. 2006. Pág. 186,187.

11. Vallejo, María del Carmen, Toxicología analítica, Departamento de toxicología y farmacia, Universidad Nacional de Colombia, Bogotá, Colombia, 1985, Pág.107-111

12. Yanggen, D.; Crissman, C.; Espinosa, P. 2003. Los plaguicidas: Impactos en producción, salud y medio ambiente en Carchi, Ecuador. Quito, CIP/INIAP. Pág. 199.

\section{Artículos Relacionados:}

13. Carmona, Jaime, Colinesterasas eritrocitaria y plasmática en trabajadores con enfermedades crónicas controladas y en usuarios de medicamentos, IATREIA n 1, volumen 19, UNIVERSIDAD DE ANTIOQUIA, Medellín- Colombia, 2006, pág 17-18

14. Cepis/OPS, Curso de Autoinstrucción en Diagnóstico, Tratamiento y Prevención de Intoxicaciones agudas causadas por plaguicidas, unidad I. Plaguicidas de tipo Organofosforados y Carbamatos, Ecuador, 2002

15. Health and Safety Executive del Reino Unido. NTP-512 Plaguicidas organofosforados http://www.mtas.es/insht/ntp/ntp_512.htm

16. Huerta, Antonio, Delgado Pedro, Plaguicidas: Neurotoxicidad y vigilancia de la salud, Centro Nacional de Medios de Protección. Sevilla-INSHT, artículo publicado en el número 8-2000, siguiendo la línea de la página Web del INSHT, páginas 4 a 14,

17. Instituto Nacional de Salud Pública de México, Sintomatología persistente en trabajadores industrialmente expuestos a plaguicidas organofosforados, 1999, Vol. 41, número 001, Cuernavaca, México, Pág. 55- 61.

18. Jiménez, Manuel, Martínez, Viria, Validación de la determinación de acetilcolinesterasa eritrocítica humana a 340 nm, REVISTA BIOMED 2000, VOLUMEN 11,San José, Costa Rica,2000 pág 161-168. http://www.uady.mx/ biomedic/rb001132.pdf

19. Milla, O, Palomino, W. "Niveles de colinesterasa sérica en agricultores de la localidad de Carapongo (Perú) y determinación de residuos de plaguicidas inhibidores de colinesterasas en frutas y hortalizas cultivadas, "Tesis para optar el título profesional de Químico farmacéutico, Lima- Perú, 2002, pág 5-6-7.

20. Organización Mundial De La Salud. Directrices para la lucha contra las intoxicaciones. Programa Internacional de Seguridad de Sustancias Químicas. Ginebra, 1998

21. OPS/OMS. Plaguicidas y salud en las Américas. Serie Ambiental 2. Washington, OPS, 1993 
22. Repetto, Robert y Baliga, Sanjay S.: Los Plaguicidas y el Sistema Inmunológico: Riesgos para la Salud Pública. World Resources Institute, marzo de 1996.

23. Sociedad De Medicina Del Trabajo, Valores Referenciales de Colinesterasas Sérica y Eritrocitaria para una población clínicamente sana de la Ciudad de Gualeguaychú, Determinación de Valores de Colinesterasa sérica y Eritrocitaria en Personas expuestas laboralmente a Plaguicidas Organofosforados, Facultad de Bromatología, Universidad Nacional de Entre Ríos, Chaco, Argentina.

24. Workshop on Epidemiological Toxicology of Pesticide Exposure (Amsterdam). Comité Científico de Pesticidas del ICOH, HUERTA, Antonio, DELGADO Pedro, PLAGUICIDAS: Neurotoxicidad y vigilancia de la salud, Centro Nacional de Medios de Protección. Sevilla-INSHT, artículo publicado en el número 8-2000, siguiendo la línea de la página Web del INSHT, páginas 4 a 14,

\section{Relatos de:}

Ingeniero agrónomo Ángel Pozo, Funcionario del Servicio Ecuatoriano de Sanidad Agropecuaria, SESA, Carchi- Ecuador.

\section{Páginas Web Investigadas:}

http://www.monografias.com/trabajos10/inhi/inhi.shtml http://www. Farm Chemicals Hanbook' 97. Pesticide Diccionary. USA.

http://www. Hemoliss con triton XIII Congreso Español de Toxicología. Granada, 22-24 de Septiembre, 1999. Rev. Toxicol. 16: 157 (1999) http://www.medilegis.com/BancoConocimiento/O/Obst-_Gin_V54_N3_inv/inv2.htm

http://www.chaco.gov.ar/SociedadMedicina/JornadasSobrePlaguicidas

facultad\%20de\%20bromatologia.htm

http:// www. NTP-512 Plaguicidas organofosforados.htm.

http://www.webmaster@estrucplan.htm

http://www.olca.cl/oca/index.htm 